\title{
Nasal airflow resistance at simulated altitude
}

\author{
J.G. Widdicombe
}

This issue includes an important paper by BARRY et al. [1] in which the changes in nasal peak inspiratory flow (NPIF), and oral peak inspiratory flow (OPIF), during acclimatized exposure to low barometric pressure (equivalent to a simulated altitude of $8000 \mathrm{~m}$, about the height of Mount Everest) of subjects in a hypobaric chamber were analysed. The changes were presumably due to hypoxia and its chemical sequelae, and influenced by the processes of acclimatization. They show that NPIF increases by a mean of $16 \%$, whereas OPIF increases by $47 \%$, and conclude that these results are consistent with a decrease in upper airways luminal calibre at altitude, correlating with the symptomatic nasal blockage and impairment of mucociliary function seen in similar conditions. The changes must be due to chronic hypoxia rather than to inhalation of cold and dry air, since environmental temperature and humidity were maintained at values close to those found at sea level. The study is important because it may point to physiological mechanisms determining airway calibre at altitude, and to changes in the airways that will influence breathing at altitude.

The authors must have confronted formidable problems in experimental design, since their subjects were confined to a hypobaric chamber for over a month, and the apparatus which could be used to assess their airway mechanics must inevitably have been limited. The authors fully justify their use of rather simple methods to assess airways' resistances. However, while these limitations can be appreciated, there are important considerations about the methodology and interpretation of results.

A clear distinction between airways' conductance, airways' resistance and airways' calibre needs to be made. The former two are reciprocally related and depend, inter alia, on the viscosity and density of the transmitted gas, and other aerodynamic features; however calibre will affect both conductance and resistance, but is not directly affected by the physical properties of the gas. NPIF and OPIF primarily reflect airways' conductance.

NPIF and OPIF are rather imprecise indications of airways' conductance. If, as the authors found, lower airways' inspiratory conductance (OPIF) is increased

GKT School of Biomedical Sciences, Human Physiology and Aerospace Medicine, Guy's Campus, London, UK.

Correspondence: J. G. Widdicombe, GKT School of Biomedical Sciences, Human Physiology and Aerospace Medicine, Shepherd's House, Guy's Campus, London Bridge, London, SE1 9RT, UK. Fax: 442082861815 at simulated altitude by a mean of $47 \%$, lower airways' inspiratory resistance would be decreased by a mean of $32 \%$. If upper airway (nasal) resistance is approximately equal to that of the lower airways [2], as seems to be the case in the authors' own experiments (their fig. 1), and if during nasal breathing the nose was to act as a constant series resistance to the lower airways, then a mechanical equivalent to Ohm's Law for electricity can be applied. This shows that NPIF (involving both upper and lower airways' conductances) should increase by $19 \%$ just because of the increase in lower airways' conductance, a value close to that obtained by the authors $(16 \%)$. It should be emphasized that this calculation is based on no change in upper airways' resistance, assuming it to be a constant, and it assumes that the method used to assess peak flows was accurately volume/flowdependent and not viscosity- or density-dependent. But the calculation suggests that, in the experimental conditions and subject to important reservations (see later), upper airways' resistance may have remained unchanged (their fig. 1), and not have increased during the chronic hypoxia. Resistance changes, or the lack of them, need to be interpreted in terms of airway calibre when gas viscosity and density are changed.

To take the authors' own values (their fig. 1), NPIF was about one-half that of OPIF, so that upper and lower airways' resistances were about equal. On the acclimatized step from simulated 0 to $8000 \mathrm{~m}$ altitude, if OPIF increased by $47 \%$ (resistance decreasing by $32 \%$ ), and if NPIF increased by $16 \%$ (resistance decreasing by $14 \%$ ), this could be explained by an increase in nasal resistance of $4 \%$, small and probably statistically insignificant.

However, although it is desirable that the study should have given more precise and physically acceptable assessments of upper and lower airways' resistances, and that the statistical analysis should have been based on such values, the authors' conclusions are valid. In this respect it is significant that on the step from 5000 to $8000 \mathrm{~m}$ altitude NPIF remained virtually constant or even decreased; if the nasal calibre had remained constant an increase in NPIF (as indeed happened to OPIF) due to the reduction in viscosity and density of the gas would have been expected. The conclusion must be that nasal calibre decreased. These results raise the question as to why hypobaric conditions reduce lower airways' resistance, and appear to decrease upper airways calibre in such a way that nasal resistance (with rarified gases) 
is approximately constant. Does some homeostatic mechanism exist to keep effective nasal resistance constant in the presence of large changes in oxygen tension and in the physical properties of the inspired gas?

The authors are undoubtedly right in their belief that the decrease in lower airways' resistance is mainly due to the mechanical characteristics of hypobaric gas. In healthy subjects breathing air at sea level a maximal bronchodilatation will only decrease lower airways' resistance by about one-half [3], not far from the $32 \%$ found by the authors. The roles of gas density and viscosity are complex, as the authors acknowledge. Both will be decreased at altitude. Laminar flow, probably predominant in the lower airways, has a direct relationship between viscosity and resistance; while turbulent airflow, probably more important in the upper airways, has a direct relationship between the square of density and resistance. The balance between laminar and turbulent airflows is set by Reynold's number, which includes the ratio of density to viscosity, but is only accurately applicable to maintained flow in long cylindrical tubes, scarcely the situation in human airways. The physics of other flow patterns is also relevant, but probably even more complicated. Only sophisticated aerodynamic analysis would allow accurate assessment of the role of the physical properties of hypobaric gas in the determination of airways' resistance. The authors' conclusions that the reduced lower airways' resistance is due to the physical properties of the gas, and that the changes in upper airways' resistance suggest a functional decrease in nasal calibre, seems sound, even if not fully quantitated.

What happens in the lower airways? With acute hypoxia, all research suggests a narrowing of the airways. Acute hypoxia reflexly contracts airways' smooth muscle [4], causes reflex mucus secretion [5], and probably airways' vasodilatation and oedema [6]. Whether these changes are modified during acclimatization to hypoxia does not seem to have been established. Pulmonary oedema is well known to be a result of chronic altitude hypoxia. The striking increase in OPIF (decrease in resistance) in the present experiments must therefore be the result of the changed physical characteristics of the inhaled gas; this conclusion is based on the assumption, already stated, that the method of measuring peak flow is either viscosity and density independent, or is corrected for their changes. Whether this change in OPIF is modulated by physiological changes in the airway wall is uncertain, but it could be supplemented by an increase in airway calibre.

The effects of inhaled gas composition on nasal physiology have been far less studied. The nasal mucosa is enclosed in a rigid bony case, so the scope for decreases in airway resistance (and increases in airway calibre) is limited. Acute hypoxia causes nasal vasoconstriction and decreased airflow resistance [7], which may be of benefit to airline passengers with nasal congestion. However acute hypoxia could also influence nasal-mucus secretion. Acclimatization to the effects of hypoxia does not seem to have been studied before in this respect, which adds to the importance of this paper. It would be valuable to have information on the time course of the changes described, to determine the role of acclimatization and to correlate them with the acclimatory changes in the control of breathing.

The paper reminds us that the neglected nose is an important target for research. Its blockage, or the lack of it, will in part determine the use of the oral pathway for breathing, which in turn may have important physiological sequelae. The processes in acclimatization may differ greatly from those in the acute state (a conclusion already well established for the control of breathing [8]). In the responses to high altitude a distinction should be made, such as the authors have done, between those due to cold and lowered humidity and those due to blood gas changes; it would be interesting to know what happens to oral and nasal resistances when the former changes are added to the latter. It would also be worthwhile to investigate the mechanisms involved in the nasal response to chronic hypoxia by the use of decongestants and antisecretants back to the hypobaric chamber? Meanwhile it is stimulating to be enthused by interesting studies and to plan other peoples' research. Incidentally, neither the paper nor this editorial considers changes in carbon dioxide tension and in $\mathrm{pH}$; that's a different can of worms!.

\section{References}

1. Barry PW, Mason NP, Richalet J-P. Nasal peak inspiratory flow at altitude. Eur Respir J 2002; 19: $16-19$.

2. Cole P. Upper respiratory airflow. In: Proctor DF, Andersen IB, eds. The nose: upper airway physiology and the atmospheric environment. Amsterdam, Elsevier Biomedical Press, 1982; pp. 167-190.

3. Widdicombe JG. Regulation of tracheobronchial smooth muscle. Physiol Revs 1963; 43: 1-37.

4. Widdicombe JG. Vagal reflexes in the airways. In: Kaliner MA, Barnes PJ, eds. The airways: neural control in health and disease. New York, Marcel Dekker, 1988; pp. 187-202.

5. Widdicombe JG, Webber SE. Airway mucus secretion. NIPS 1990; 5: 2-5.

6. Godden DJ. Reflex and nervous control of the tracheobronchial circulation. Eur Respir J 1990; 3: 602-607s.

7. Eccles R. Neurological and pharmacological considerations. In: Proctor DF, Andersen IP, eds. The nose: upper airway physiology and the atmospheric environment. Amsterdam, Elsevier Biomedial Press, 1982; pp. 191-214.

8. Forster HV, Dempsey JA. Ventilatory adaptations. In: Hornbein TF, ed. Regulation of breathing. New York, Marcel Dekker, 1981; pp. 845-904. 